\title{
Transient evoked otoacoustic emissions and auditory brainstem response in infants with perinatal asphyxia
}

\author{
Georgea Espindola Ribeiro ${ }^{\text {a, *, }}$, Daniela Polo Camargo da Silva a, Jair Cortez Montovani ${ }^{b}$ \\ a Botucatu Medical School, São Paulo State University (UNESP), Brazil \\ b Department of Ophthalmology, Otorhinolaryngology and Head and Neck Surgery, Botucatu Medical School, São Paulo State University (UNESP), Brazil
}

\section{A R T I C L E I N F O}

\section{Article history:}

Received 7 April 2016

Received in revised form

19 July 2016

Accepted 11 August 2016

Available online 15 August 2016

\section{Keywords:}

Neonatal screening

Hearing loss

Newborn infant

Electrophysiology

\begin{abstract}
A B S T R A C T
Objective: The objective of this study was to verify the effects of perinatal asphyxia on different parts of the auditory system.

Methods: This was a non-concurrent cohort study conducted on a fixed population in a tertiary public hospital. Participants included 181 infants born at term who underwent the transient evoked otoacoustic emission test as a part of a neonatal hearing screening program, with a "pass" result in both ears, and by auditory brainstem response testing. The infants were divided into 3 groups: G1, 20 infants who had perinatal asphyxia; G2, 111 infants with an Apgar score lower than 4 in the first minute and/or lower than 6 in the fifth minute (called "low Apgar" at birth); and G3, 50 infants with first- and fifth-minute Apgar scores $\geq 7$.

Results: The signal-to-noise ratio of transient evoked otoacoustic emissions were greater in G3 compared with G1 and G2 at $4 \mathrm{kHz}$ frequency for males. An increased latency of waves I and III in the auditory brainstem response of male infants in G1 was observed.

Conclusion: This study demonstrated that alterations occurred in both the cochlear and the neural components in male infants who had perinatal asphyxia.
\end{abstract}

(c) 2016 Elsevier Ireland Ltd. All rights reserved.

\section{Introduction}

Hearing is essential for language development. A prerequisite for hearing acquisition and development is the anatomical and physiological integrity of the neurologic and auditory system [1,2].

Therefore, all neonates should undergo a hearing assessment soon after birth. Investigation of auditory abnormalities may allow early diagnosis and treatment to minimize impacts on language development $[3,4]$.

Some events may increase the chances for hearing loss. One such risk indicator is perinatal asphyxia, which is defined as an injury experienced by the neonate when there is significant peripartum hypoperfusion and decreased oxygen delivery resulting from different etiologies [5,6].

Hearing tests currently conducted on newborns include transient evoked otoacoustic emissions (TEOAE) and auditory

\footnotetext{
* Corresponding author. Department of Ophthalmology, Otorhinolaryngology and Head and Neck Surgery, Botucatu Medical School, São Paulo State University (UNESP), Distrito de Rubião Junior s/n, 18618-970, Brazil.

E-mail address: georgea_espindola@hotmail.com (G.E. Ribeiro).
}

brainstem response $(\mathrm{ABR})$. The first evaluates the integrity of the outer hair cells $(\mathrm{OHC})[7,8]$. The second checks the integrity of the auditory pathway and estimates the auditory threshold [9].

Infants with risk indicators for hearing loss have more abnormal results in those tests. Studies in neonates with perinatal asphyxia demonstrate that hearing loss may occur in an isolated manner, at cochlear as well as neural portions. Many authors have demonstrated that these effects may be transient [10]. Detailed investigation of these structures in the newborn population enables further understanding of the true damage caused by this injury.

The objective of the study was to verify the effect of perinatal asphyxia in different parts of the auditory system.

\section{Methods}

The Ethics Committee of the hospital approved this study (process number 156/2012).

Data were collected from January 2014 to December 2015.

This was a non-concurrent cohort study on fixed population; therefore, exclusion criteria were not required. The inclusion criteria were: a) having been born in the hospital of the study; b) 
Table 1

Population characteristics.

\begin{tabular}{llll}
\hline Group/Variables & $\mathrm{G} 1(\mathrm{n}=20)$ & $\mathrm{G} 2(\mathrm{n}=111)$ & $\mathrm{G} 3(\mathrm{n}=50)$ \\
\hline Female/Male & $8(40 \%) / 12(60 \%)$ & $38(34 \%) / 73(66 \%)$ & $26(52 \%) / 24(48 \%)$ \\
Gestational age $^{\mathrm{a}}$ & $39(37-42)$ & $39(37-42)$ & $38(37-41)$ \\
Birth weight $^{\mathrm{a}}$ & $3315(26304950)$ & $3250(2520-4465)$ & $3302(2555-4190)$ \\
\hline
\end{tabular}

a Summary as mean (minimum and maximum) gestational age and weight at birth.

gestational age $\geq 37$ weeks; c) normal external auditory canal and tympanic membrane, as assessed by an ENT physician specializing in child hearing loss; d) a "pass" response in both ears on the TEOAE test, and ABR with all components identified; e) informed consent signed by the parents.

The population was divided into three groups:

- Group 1 ( $\mathrm{G} 1, \mathrm{n}=20)$, infants with a medical diagnosis of asphyxia who presented with at least two of the following parameters: $\mathrm{pH} \leq 7.20$ in the umbilical vein; Apgar score $\leq 6$ at the fifth minute; and fraction of inspired oxygen $\geq 0.4$ needed to achieve oxygen saturation $\geq 86 \%$ at birth [11].

- Group 2 (G2, n =111), infants with an Apgar score $<4$ in the first minute and/or $<6$ in the fifth minute (called "low Apgar").

- Group 3 (G3, n=50), infants with first- and fifth-minute Apgar scores $>7$ ("control").

The TEOAE tests were performed with OtoRead (Interacoustics), a portable otoacoustic emissions instrument used in hearing screening programs. To obtain the responses the probe was coupled to the external ears of the newborn, preferably during the neonate's physiological sleep or when he or she was calm and in a quiet room. All neonates were evaluated after the 48th hour of life.

For analysis, the parameter "pass/refer" was used, as described in the equipment instructions. Click stimulus, intensity $83 \mathrm{~dB}$ sound pressure level (SPL), and six frequency bands were evaluated (1.5; $2 ; 2.5 ; 3 ; 3.5$ and $4 \mathrm{kHz}$ ). Parameters related to whole wave reproducibility and total echo power are established into the equipment, and only perfect evaluation may achieve a "PASS" result. Values considered as PASS were: emissions present with a signal-to-noise ratio of $6 \mathrm{~dB}$ in at least three consecutive frequency bands, including $4 \mathrm{kHz}$.

The ABR test was conducted at one month of age, using the EP15 system (Eclipse/Interacoustics) in a quiet room. After cleaning the skin with an abrasive product, positive surface electrodes (Neuroline) were fixed to the Fz, and negative surface electrodes were fixed to M1 and M2. The ground lead was placed on Fpz. The stimulus was presented by means of an insertion phone (ER 3A), with monoaural stimulation, using filtered clicks (between 0.1 and $3 \mathrm{kHz}$ ) lasting $100 \mu \mathrm{s}$, rarefied polarity, at an intensity of $80 \mathrm{~dB}$ NPS. 1024 clicks were provided, at a presentation rate of 20.1 clicks/ second, with an analysis time of $15 \mathrm{~ms}$, and were repeated to confirm wave reproduction. Electrode impedance was maintained below 5 Kohms.

The groups were compared in relation to gender-stratified results using the non-parametric Kruskal-Wallis test, followed by the Dunn test for multiple comparisons. Normal distribution of the outcomes was rejected using the Shapiro-Wilk test. Differences were considered statistically significant when $\mathrm{p}<0.05$. Analysis was performed using SPSS v21.0 software.

With regard to sample size, considering an error type $\mathrm{I}=0.05$, the absence of confounding factors and the symmetry of results, our estimates indicate that the test power may be less than 0.8 .

\section{Results}

Population data characteristics are described in Table 1.

In our first analysis, we observed that gender influenced the TEOAE and ABR results. To better address the research question, we separated our data by gender.

When comparing the TEOAE signal-to-noise ratio, we observed no significant differences between the groups of females. In males, however, the signal-to-noise ratios were greater in $\mathrm{G} 3$ as compared to $\mathrm{G} 1$ and $\mathrm{G} 2$ at the $4 \mathrm{kHz}$ frequency, in both ears (Tables 2 and 3).

Furthermore we observed no differences when comparing ABR's absolute and interpeak latencies in the female groups, but we found increased absolute latencies for waves I and III in G1 male infants, in both ears, using the Kruskal-Wallis test. However, when we applied the Dunn test to differentiate the groups, we found no statistical differences in the analysis of wave III for the left ear (Tables 4 and 5).

\section{Discussion}

This study aimed to verify the effects of perinatal asphyxia in different parts of the auditory system, specifically, in the cochlea as well as in the auditory pathway.

In this study, newborn hearing assessments were performed twice; the first was conducted by TEOAE at 48 h following birth, and the second was conducted by ABR at one month of age. The time between the tests was necessary because they are performed in different sites. The TEOAE was performed in the nursery, and the ABR was performed in the hospital diagnostic center. However, all of the newborns included in the study had normal external auditory canals and tympanic membranes, as assessed by an ENT physician specializing in child hearing loss prior to both evaluations.

Another observation concerns the numbers of subjects in the different groups. We had a greater number of newborns with only a low Apgar score (G2) and fewer with asphyxia (G1) because it is a normal occurrence in the sample. We included 50 newborns in the control group, representing all of the infants whose parents agreed to participate in the research during the recruitment period.

The criteria used to characterize the TEOAE findings usually are based on a "pass/refer" response [12]. However, analysis of the

Table 2

Comparison between groups in relation to the response signal to noise ratio of TEOAE in female infants.

\begin{tabular}{|c|c|c|c|c|c|c|c|c|c|c|c|}
\hline & & \multicolumn{3}{|c|}{$\mathrm{G} 1(\mathrm{n}=8)$} & \multicolumn{3}{|c|}{$\mathrm{G} 2(\mathrm{n}=38)$} & \multicolumn{3}{|c|}{$\mathrm{G} 3(\mathrm{n}=26)$} & \multirow[t]{2}{*}{$P$} \\
\hline & & Med & Min & Max & Med & Min & Max & Med & Min & Max & \\
\hline \multirow{3}{*}{ Right ear } & $3.0 \mathrm{kHz}$ & 9.5 & 6.0 & 19.0 & 12.0 & 6.0 & 23.0 & 12.5 & 6.0 & 25.0 & 0.131 \\
\hline & $3.5 \mathrm{kHz}$ & 13.5 & 7.0 & 25.0 & 14.5 & 6.0 & 25.0 & 16.0 & 6.0 & 25.0 & 0.861 \\
\hline & $4.0 \mathrm{kHz}$ & 13.5 & 7.0 & 25.0 & 14.5 & 6.0 & 25.0 & 13.5 & 6.0 & 27.0 & 0.916 \\
\hline \multirow[t]{3}{*}{ Left ear } & $3.0 \mathrm{kHz}$ & 7.5 & 6.0 & 19.0 & 10.5 & 6.0 & 25.0 & 18.5 & 6.0 & 27.0 & 0.135 \\
\hline & $3.5 \mathrm{kHz}$ & 14.5 & 6.0 & 30.0 & 14.0 & 6.0 & 27.0 & 17.0 & 6.0 & 25.0 & 0.701 \\
\hline & $4.0 \mathrm{kHz}$ & 15.5 & 6.0 & 23.0 & 14.0 & 6.0 & 27.0 & 16.5 & 6.0 & 27.0 & 0.677 \\
\hline
\end{tabular}

Med: median, Min: minimum, Max: maximum, ( $<<0.05$; Kruskal-Wallis test). 
Table 3

Comparison between groups in relation to response signal to noise ratio of TEOAE in male infants.

\begin{tabular}{|c|c|c|c|c|c|c|c|c|c|c|c|}
\hline & & \multicolumn{3}{|c|}{$\mathrm{G} 1(\mathrm{n}=12)$} & \multicolumn{3}{|c|}{$\mathrm{G} 2(\mathrm{n}=73)$} & \multicolumn{3}{|c|}{$\mathrm{G} 3(\mathrm{n}=24)$} & \multirow[t]{2}{*}{$P$} \\
\hline & & Med & Min & Max & Med & Min & Max & Med & Min & Max & \\
\hline \multirow{3}{*}{ Right ear } & $3.0 \mathrm{kHz}$ & 7.0 & 6.0 & 17.0 & 11.0 & 6.0 & 29.0 & 9.0 & 6.0 & 22.0 & 0.110 \\
\hline & $3.5 \mathrm{kHz}$ & 9.0 & 6.0 & 26.0 & 13.0 & 6.0 & 29.0 & 12.0 & 6.0 & 28.0 & 0.411 \\
\hline & $4.0 \mathrm{kHz}$ & 8.0 & 6.0 & 23.0 & 12.0 & 6.0 & 29.0 & 16.5 & 6.0 & 25.0 & $0.012^{\mathrm{a}}$ \\
\hline \multirow[t]{3}{*}{ Left ear } & $3.0 \mathrm{kHz}$ & 8.5 & 6.0 & 16.0 & 10.0 & 6.0 & 27.0 & 10.0 & 6.0 & 24.0 & 0.482 \\
\hline & $3.5 \mathrm{kHz}$ & 13.5 & 6.0 & 22.0 & 12.0 & 6.0 & 26.0 & 15.5 & 6.0 & 26.0 & 0.162 \\
\hline & $4.0 \mathrm{kHz}$ & 12.0 & 6.0 & 22.0 & 11.0 & 6.0 & 29.0 & 15.5 & 8.0 & 26.0 & $0.009^{\mathrm{b}}$ \\
\hline
\end{tabular}

Med: median, Min: minimum and Max: maximum, ( $<<0.05$; Kruskal-Wallis test).

( $\mathrm{p}<0.05$; Dunn's multiple comparison test).

a $\mathrm{G} 3>\mathrm{G} 1$.

b $\mathrm{G} 3>\mathrm{G} 2$.

Table 4

Comparison of ABR absolute and interpeak latencies in female infants.

\begin{tabular}{|c|c|c|c|c|c|c|c|c|c|c|c|}
\hline & & \multicolumn{3}{|c|}{$\mathrm{G} 1(\mathrm{n}=8)$} & \multicolumn{3}{|c|}{$\mathrm{G} 2(\mathrm{n}=38)$} & \multicolumn{3}{|c|}{$\mathrm{G} 3(\mathrm{n}=26)$} & \multirow[t]{2}{*}{$P$} \\
\hline & & Med & Min & Max & Med & Min & Max & Med & Min & Max & \\
\hline \multirow[t]{6}{*}{ Right ear } & I & 1.4 & 1.3 & 1.6 & 1.4 & 1.3 & 1.6 & 1.4 & 1.3 & 1.8 & 0.363 \\
\hline & III & 4.0 & 3.8 & 4.5 & 3.9 & 3.6 & 4.7 & 4.0 & 3.7 & 4.7 & 0.448 \\
\hline & $\mathrm{V}$ & 6.2 & 5.8 & 6.5 & 6.2 & 5.8 & 6.9 & 6.1 & 5.7 & 6.9 & 0.992 \\
\hline & I-III & 2.6 & 2.2 & 3.0 & 2.5 & 2.2 & 3.1 & 2.6 & 2.2 & 3.4 & 0.460 \\
\hline & III-V & 2.1 & 1.9 & 2.3 & 2.2 & 1.9 & 2.8 & 2.1 & 1.8 & 2.5 & 0.246 \\
\hline & $\mathrm{I}-\mathrm{V}$ & 4.8 & 4.2 & 5.0 & 4.7 & 4.3 & 5.4 & 4.7 & 4.1 & 5.6 & 0.995 \\
\hline \multirow[t]{6}{*}{ Left ear } & I & 1.5 & 1.3 & 1.7 & 1.4 & 1.3 & 1.7 & 1.4 & 1.3 & 1.7 & 0.073 \\
\hline & III & 4.1 & 3.9 & 4.5 & 4.0 & 3.7 & 4.8 & 4.0 & 3.6 & 4.6 & 0.248 \\
\hline & V & 6.2 & 5.9 & 6.6 & 6.2 & 5.8 & 6.9 & 6.1 & 5.7 & 6.9 & 0.882 \\
\hline & I-III & 2.6 & 2.3 & 2.8 & 2.6 & 2.2 & 3.1 & 2.6 & 2.3 & 3.3 & 0.681 \\
\hline & III-V & 2.1 & 2.0 & 2.2 & 2.2 & 1.9 & 2.8 & 2.1 & 1.8 & 2.5 & 0.115 \\
\hline & $\mathrm{I}-\mathrm{V}$ & 4.7 & 4.3 & 4.9 & 4.7 & 4.1 & 5.3 & 4.8 & 2.5 & 5.4 & 0.657 \\
\hline
\end{tabular}

Med: median, Min: minimum, Max: maximum, ( $<0.05$; Kruskal-Wallis test).

signal-to-noise ratio for TEOAE, which is the intensity of the emission originated from the cochlea, as a function of the noise expressed in decibels sound pressure level (dB SPL), can provide additional information on the condition and function of the inner ear, particularly on the OHC [13]. This type of analysis is possible only for infants who display "pass" responses on the test.

Several studies raise the hypothesis that females show a greater signal-to-noise ratio in the TEOAE test and smaller latencies in the ABR $[14,15]$. For this reason, we conducted our analyses separately, by gender, for both tests.

We noted that male infants who had perinatal asphyxia had a lower signal-to-noise ratio in the TEOAE test at $4 \mathrm{kHz}$ frequency, but in females, no difference was found. These data were similar to those reported by other authors [14], who also observed a significantly lower signal to noise ratio in the TEOAE of male infants, suggesting that male infants are more susceptible to damage in the OHC after asphyxia.

The literature is unclear regarding the clinical relevance of this

Table 5

Comparison of ABR absolute and interpeak latencies in male infants.

\begin{tabular}{|c|c|c|c|c|c|c|c|c|c|c|c|}
\hline & & \multicolumn{3}{|c|}{$\mathrm{G} 1(\mathrm{n}=12)$} & \multicolumn{3}{|c|}{$\mathrm{G} 2(\mathrm{n}=73)$} & \multicolumn{3}{|c|}{$\mathrm{G} 3(\mathrm{n}=24)$} & \multirow[t]{2}{*}{$P$} \\
\hline & & Med & Min & Max & Med & Min & Max & Med & Min & Max & \\
\hline \multirow[t]{6}{*}{ Right ear } & I & 1.5 & 1.3 & 1.9 & 1.4 & 1.2 & 1.7 & 1.4 & 1.3 & 1.7 & $0.005^{\mathrm{a}}$ \\
\hline & III & 4.2 & 3.8 & 4.9 & 4.0 & 3.6 & 4.5 & 4.1 & 3.6 & 4.5 & $0.025^{\mathrm{b}}$ \\
\hline & V & 6.5 & 6.0 & 7.1 & 6.3 & 5.6 & 6.9 & 6.4 & 6.0 & 6.8 & 0.184 \\
\hline & I-III & 2.8 & 2.3 & 3.1 & 2.6 & 2.2 & 3.1 & 2.7 & 2.3 & 3.1 & 0.066 \\
\hline & III-V & 2.2 & 1.8 & 2.5 & 2.3 & 1.8 & 2.9 & 2.3 & 2.0 & 2.6 & 0.837 \\
\hline & $\mathrm{I}-\mathrm{V}$ & 5.0 & 4.6 & 5.6 & 4.9 & 4.3 & 5.4 & 5.0 & 4.5 & 5.4 & 0.777 \\
\hline \multirow[t]{6}{*}{ Left ear } & I & 1.5 & 1.4 & 1.9 & 1.4 & 1.2 & 1.7 & 1.4 & 1.2 & 1.7 & $0.011^{\mathrm{c}}$ \\
\hline & III & 4.2 & 3.8 & 4.6 & 4.0 & 3.6 & 4.7 & 4.1 & 3.6 & 4.6 & $0.030^{\mathrm{d}}$ \\
\hline & V & 6.5 & 6.0 & 7.0 & 6.3 & 5.6 & 6.8 & 6.4 & 6.0 & 6.8 & 0.217 \\
\hline & I-III & 2.7 & 2.4 & 3.0 & 2.6 & 2.3 & 3.2 & 2.7 & 2.3 & 3.2 & 0.056 \\
\hline & III-V & 2.2 & 1.9 & 2.6 & 2.2 & 1.8 & 2.9 & 2.2 & 1.9 & 2.6 & 0.577 \\
\hline & $\mathrm{I}-\mathrm{V}$ & 5.0 & 4.6 & 5.4 & 4.9 & 4.3 & 5.4 & 5.0 & 4.6 & 5.3 & 0.604 \\
\hline
\end{tabular}

Med: median, Min: minimum, Max: maximum, $(\mathrm{p}<0.05$; Kruskal-Wallis test)

( $p<0.05$; Dunn's test for multiple comparisons).

${ }^{\text {a }} \mathrm{G} 2, \mathrm{G} 3<\mathrm{G} 1$.

b $\mathrm{G} 2<\mathrm{G} 1$.

c $\mathrm{G} 2, \mathrm{G} 3<\mathrm{G} 1$.

d $\mathrm{G} 1=\mathrm{G} 2=\mathrm{G} 3$. 
finding. Few studies have verified the value of the TEOAE signal-tonoise ratio in routine auditory assessment of infants or investigated it in the occurrence of risk indicators for hearing loss.

Studies have demonstrated that a decreased signal-to-noise ratio may be an indication of cochlear abnormalities, regardless of "pass" result on the TEOAE test [16]. This observation justifies its analysis in the many injuries that can affect the pediatric population.

In this study, the significantly reduced signal-to-noise ratio at the $4 \mathrm{kHz}$ frequency may be an indication of cochlear damage with potential later consequences. Audibility in this region is directly related to the recognition of acute phonemes, with impacting their acquisition and reproduction [17].

Many authors report low TEOAE "pass" rates in infants with perinatal asphyxia, strongly reinforcing the hypothesis of injury to the OHC caused by tissue hypoxia. Depending on the level of asphyxia, injury may not be detected by the TEOAE "pass/refer" criteria following only one auditory assessment $[18,19]$.

Data analysis based not only on the "pass/refer" criteria but also on TEOAE signal-to-noise findings enables a more precise audiological diagnosis, in order to refine normality criteria adopted by neonatal hearing screening programs.

In the ABR test, we observed increased latencies in the I and III waves, in both ears, for male infants with asphyxia. These increased latencies may relate to a hypoxia-induced reduction in electrical conduction, consequent to a neural maturation delay or a loss in structures of the brainstem auditory pathway [20].

The true effects of perinatal asphyxia in the auditory pathways and whether it is temporary or not remain controversial issues. Researchers have observed increased ABR thresholds in animals immediately following asphyxia; upon retesting, hours later, the responses fell within normal patterns [21]. Others have observed that rats with asphyxia at birth showed increased ABR absolute and interpeak latencies, but they did not retest the animals [22]. Similar findings have been described by Tomimatsu et al. [23], who noted significant increases in the III-V and I-V interpeak latencies in ABR tests of rats with asphyxia, as compared to control rats.

Jiang et al. (2001) [24] reported an increase in the V wave latencies and $\mathrm{I}-\mathrm{V}$ and III-V interpeak findings in infants with perinatal asphyxia. The researchers attributed those observations to the fact that damage incurred from a lack of oxygen can cause alterations in the brainstem auditory pathway.

Our observations demonstrate that the alterations occur on the auditory nerve (I wave) and cochlear nucleus (III wave). They show a statistical trend of injury in both the cochlea and the auditory nerve, which is most prominent in our analysis of ABR wave latencies in males. Few authors have reported such differences between genders. Pinto and Matas (2007) [25] observed faster cochlear responses in females, which would influence brainstem responses, even affecting subjects with low Apgar scores or perinatal asphyxia.

Finally, our data suggest that infants who had perinatal asphyxia and have "pass" results in the TEOAE and presence of waves in the ABR should be followed and undergo routine audiological assessment, in order to improve management and treatment.

\section{Conclusion}

This study demonstrated alterations in both the cochlear and the neural components in male infants who had perinatal asphyxia.

Additionally, evaluation of the response signal-to-noise ratio in the otoacoustic emission test provided additional information for the audiological assessment of this population, indicating damage to the integrity of the peripheral auditory system. This damage was not possible to detect simply using the TEOAE "pass/refer" criteria.

\section{Acknowledgments}

We thank the children and their guardians for participating in the research.

\section{References}

[1] C. Yoshinaga-Itano, A.L. Sedey, D.K. Coulter, A.L. Mehl, Language of early-and later-identified children with hearing loss, Pediatrics 102 (1998) 1161-1171.

[2] Joint Committee on Infant Hearing, Year 2000 position statement: principles and guidelines for early detection and intervention programs, Pediatrics 106 (2000) 798-817.

[3] M. Maqbool, B.A. Najar, I. Gattoo, et al., Screening for hearing impairment in high risk neonates: a hospital based study, J. Clin. Diagn. Res. 9 (2015) 18-21.

[4] D.C. Malta, E.C. Duarte, M.F. Almeida, et al., List of avoidable causes of deaths due to interventions of the Brazilian health system, Epidemiol. Serv. Saude 16 (2007) 233-244.

[5] T. Revakova, A. Vasilenkova, E. Ujhazy, et al., Impact of asphyxia on red blood cell folate concentration levels in newborns, Bratisl. Lek. Listy 116 (2015) $417-421$.

[6] P. Mincarone, C.G. Leo, S. Sabina, Evaluating reporting and process quality of publications on UNHS: a systematic review of programmes, BMC Pediatr. 22 (2015) 15-86.

[7] J.S. Gravel, K.R. White, J.L Johnson, et al. A multisite study to examine the efficacy of the otoacoustic emission/automated auditory brainstem response newborn hearing screening protocol: recommendations for policy, practice, and research, Am. J. Audiol. 14 (2005) 217-228.

[8] W.D. Eiserman, D.M. Hartel, L. Shisler, et al., Using otoacoustic emissions to screen for hearing loss in early childhood care settings, Int. J. Pediatr. Otorhinolaryngol. 72 (2008) 475-482.

[9] T. Okhravi, S. Tarvij Eslami, A. Hushyar Ahmadi, et al., Evaluation of auditory brain stems evoked response in newborns with pathologic hyperbilirubinemia in mashhad, Iran, Iran. Red. Crescent Med. J. 4 (2015) 18288.

[10] R. Beswick, C. Driscoll, J. Kei, et al., Which risk factors predict postnatal hearing loss in children? J. Am. Acad. Audiol. 24 (2013) 205-213.

[11] G. Buonocore, S. Perrone1, M. Longiniet, et al., Oxidative stress in preterm neonates at birth and on the seventh day of life, Pediatr. Res. 52 (2002) 46-49.

[12] R.M. Onoda, M.F. Azevedo, A.M. Santos, Neonatal Hearing Screening: failures, hearing loss and risk indicators, Braz. J. Otorhinolaryngol. 77 (2011) 775-783.

[13] D. Aidan, P. Lestang, P. Avan, et al., Characteristics of transient-evoked otoacoustic emissions (TEOEs) in neonates, Acta Otolaryngol. 117 (1997) 25-30.

[14] M.C.A. Basseto, B.M. Chiari, M.F. Azevedo, Transient evoked otoacoustic emissions (TEOAE): response amplitude in term and pre-term neonates, Rev. Bras. Otorrinolaringol. 69 (2003) 84-92.

[15] R.M.G. Angrisani, A.P.D. Bautzer, C.G. Matas, et al., Auditory brainstem response in neonates: influence of gender and weight/gestational age ratio, Rev. Paul. Pediatr. 31 (2013) 494-500.

[16] P.A. Bonfils, J.P. Piron, A. Uziel, et al., Correlative study of evoked otoacoustic emission properties and audiometric thresholds, Arch. Otorhinolaryngol. 245 (1988) 53-56.

[17] O. Segal, D. Houston, L. Kishon-Rabin, Auditory discrimination of lexical stress patterns in hearing-impaired infants with cochlear implants compared with normal hearing: influence of acoustic cues and listening experience to the ambient language, Ear Hear 37 (2016) 225-234.

[18] K. Wroblewska-Seniuk, K. Chojnacka, B. Pucher, et al., The results of newborn hearing screening by means of transient evoked otoacoustic emissions, Int. J. Pediatr. Otorhinolaryngol. 69 (2005), 1351-2137.

[19] S. Coenraada, A. Goedegeburea, J.B. van Goudoever, et al., Risk factors for sensorineural hearing loss in NICU infants compared to normal hearing NICU controls, Eur. Arch. Otorhinolaryngol. 267 (2010) 1531-1537.

[20] M.P. Gorga, J.R. Kaminski, K.L. Beauchaine, et al., Auditory brainstem responses from children three months to three years of age: normal patterns of response II, J. Speech. Hear. Res. 32 (1989) 281-288.

[21] Y. Cycowicz, M. Schmuel, S. Freeman, et al., Perinatal hypoxia and auditory brainstem response thresholds: no evidence of permanent hearing loss, Hear. Res. 33 (1988), 239-224.

[22] A.L. Smit, M. Seehase, R.J. Stokroos, et al., Functional impairment of the auditory pathway after perinatal asphyxia and the short-term effect of perinatal propofol anesthesia in lambs, Pediatr. Res. 74 (2013) 34-38.

[23] T. Tomimatsu, H. Fukuda, M. Endoh, et al., Effects of neonatal hypoxicischemic brain injury on skilled motor tasks and brainstem function in adult rats, Brain. Res. 926 (2002) 108-117.

[24] Z.D. Jiang, D.M. Brosi, A.R. Wilkinson, Comparison of brainstem auditory evoked responses recorded at different presentation rates of clicks in term neonates after asphyxia, Acta Paediatr. 90 (2001) 1416-1420.

[25] F.R. Pinto, C.G. Matas, A comparison between hearing and tone burst electrophysiological thresholds, Rev. Bras. Otorrinolaringol. 73 (2007) 513-522. 